\title{
Strategic Management in Marketing: A Game Theoretic Approach
}

\author{
Vinod Jangid ${ }^{\mathrm{a}}$, Gaurav Sharma ${ }^{\mathrm{b}}$ and ${ }^{*}$ Ganesh Kumar $^{\mathrm{c}}$ \\ ${ }^{a}$ Research Scholar, Department of Mathematics , University of Rajasthan, Jaipur-302004, (India) \\ ${ }^{\mathrm{b}}$ Department of Mathematics, Acharya Narendra Dev College University of Delhi, (India) \\ 'Assistant Professor, Department of Mathematics , University of Rajasthan, Jaipur-302004, (India) \\ *Corresponding author email ID: ganeshmandha1988@gmail.com
}

\begin{abstract}
In marketing, a real-world dilemma emerging between two rivals, McDonald's and Burger King, is investigated. Both firms use three strategies: discounted pricing, status quo, and aggressive commercial. In such cases, ambiguity is a determining factor. To deal with confusion in payoffs, octagonal fuzzy numbers are used. To rank fuzzy numbers, the average of odd positions, average of even positions, and quartile deviations are used. To solve the reduced modelled two competitors zero sum fuzzy matrix games, the proposed ranking methods are used. Finally, the findings are compared to current approaches that are quite similar to the proposed approach.
\end{abstract}

Keywords: Marketing Management, Fuzzy Matrix Games, Octagonal Fuzzy Numbers, Ranking

\section{Introduction}

Uncertain parameters are known as fuzzy numerical data, and they can be expressed by a variety of fuzzy numbers. Zadeh (1965) developed this principle and applied it to real-world problems. McGvire and Staelin examined and established chain equilibrium principles (1983). Milgroom and Roberts (1986) conducted extensive research on pricing and advertisement signals of product quality. Mitchell and Hustad pioneered product screening techniques (1981). Nadia and Pishkoohi investigated traffic management issues in a fuzzy environment (2018). Bortolan and Degani (1985) developed a variety of methods for fuzzy set applications that can be used to rank different preferences developed by Watson, Yager, Chang, Adamo, Bass, Skwakernaak, Baldwins Giuld, Jain Dubois, and Prade. Butnariu (1978) defined N-person fuzzy games. Using a theoretical approach to fuzzy mathematics, Cevikel and Ahlataiuglu (2009) published some new solution procedures for fuzzy matrix games. They spoke about the case of linearity in fuzzy payoffs. Following the formulation of the linear programming problem, Sakawa's method was used to find the solution. Christi and Kalpana (2016) used trapezoidal fuzzy numbers as payoffs in fuzzy matrix games and obtained optimal value results using an average weighted approach. Delgado and Verdegay developed fuzzy ordered relations within fuzzy numbers (1988). In a fuzzy setting, Liou and Wang (1992) established some alternative approaches. Yuan (1991) developed a ranking system with integral value and evaluated it using four evolutionary criteria. These four requirements are fuzzy ordering rationality, robustness, fuzzy choice, representation, and distinguishability. Sharma and Kumar (2016) used the idea of fuzzy numbers in game theory to predict election outcomes. Malini and Kennedy also addressed OFN and their activities (2013). Malini and Anthanarajan (2016) used OFN to develop fuzzy transportation problems.

The current paper is structured as follows: section 2 contains some simple preliminaries. Section 3 discusses basic terminology for two-person zero-sum games in a fuzzy environment. Section 4 investigates current methods for ranking fuzzy numbers. Section 5 presents our proposed process. In section 6 , suggested methods are applied to find optimal strategies for two firms, and numerical examples are given. Section 7 discusses graphical representations of performance, and Section 8 provides conclusions.

\section{Preliminaries}

Definition 2.1 "Let $X$ be the universe whose generic elements are denoted by $\mathrm{x}$. A fuzzy set $\widetilde{A}$ in $X$ is characterized by its membership function $\mu_{\widetilde{A}}: X \rightarrow[0,1]$ and $\mu_{\widetilde{A}}(x)$ is interpreted as the degree of membership of element $\mathrm{x}$ in the fuzzy set $\widetilde{\mathrm{A}}$ for each $\mathrm{X} \in \mathrm{X}$. Thus a fuzzy set $\widetilde{\mathrm{A}}$ in $\mathrm{X}$ can also be represented as $\widetilde{\mathrm{A}}=$ $\left\{\left(\mathrm{x}, \mu_{\widetilde{\mathrm{A}}}(\mathrm{x})\right)\right\}$. The value zero is used to represent complete non-membership, the value one is used to represent complete membership and the values between zero and one are used to represent intermediate degrees of membership."

Definition 2.2"A fuzzy set $\widetilde{\mathrm{A}}$ in $\mathrm{R}$ is called a fuzzy number if it satisfies the following axioms:

(i) There exist at least one $x_{0} \in R$ with $\mu_{\widetilde{A}}\left(x_{0}\right)=1$ 
(ii) $\mu_{\widetilde{A}}(\mathrm{x})$ is piecewise continuous.

(iii) A must be normal and convex."

Definition 2.3 "A fuzzy number $\widetilde{A}$ is a normal octagonal fuzzy number denoted by $\widetilde{\mathrm{A}}=\left(\mathrm{a}_{1}, \mathrm{a}_{2}, \mathrm{a}_{3}, \mathrm{a}_{4}, \mathrm{a}_{5}, \mathrm{a}_{6}, \mathrm{a}_{7}, \mathrm{a}_{8}\right)$ where $\mathrm{a}_{1} \leq \mathrm{a}_{2} \leq \mathrm{a}_{3} \leq \mathrm{a}_{4} \leq \mathrm{a}_{5} \leq \mathrm{a}_{6} \leq \mathrm{a}_{7} \leq \mathrm{a}_{8}$ are real numbers and its membership function $\mu_{\widetilde{A}}(x)$ is given by

$$
\mu_{\widetilde{A}}(x)=\left\{\begin{array}{ll}
\frac{0}{k}\left(\frac{x-a_{1}}{a_{2}-a_{1}}\right) & ; x<a_{1} \\
k & ; a_{1} \leq x \leq a_{2} \\
\left\{k+(1-k)\left(\frac{x-a_{3}}{a_{4}-a_{3}}\right)\right\} & ; a_{2} \leq x \leq a_{3} \\
1 & ; a_{3} \leq x \leq a_{4} \\
\left\{k+(1-k)\left(\frac{a_{6}-x}{a_{6}-a_{5}}\right)\right\} & ; a_{4} \leq x \leq a_{5} \\
k & ; a_{5} \leq x \leq a_{6} \\
k\left(\frac{a_{8}-x}{a_{8}-a_{7}}\right) & ; a_{6} \leq x \leq a_{7} \\
0 & ; a_{7} \leq x \leq a_{8}
\end{array}\right\}
$$

Definition 2.4 "Let $\widetilde{A}=\left(a_{1}, a_{2}, a_{3}, a_{4}, a_{5}, a_{6}, a_{7}, a_{8}\right)$ be an octagonal fuzzy number, then the $\alpha$-cut is defined as

$$
\widetilde{\mathrm{A}}_{\alpha}=\left\{\begin{array}{cc}
{\left[\mathrm{a}_{1}+\frac{\alpha}{\mathrm{k}}\left(\mathrm{a}_{2}-\mathrm{a}_{1}\right), \mathrm{a}_{8}-\frac{\alpha}{\mathrm{k}}\left(\mathrm{a}_{8}-\mathrm{a}_{7}\right)\right]} & ; \alpha \in[0, \mathrm{k}] \\
{\left[\mathrm{a}_{3}+\left(\frac{\alpha-\mathrm{k}}{1-\mathrm{k}}\right)\left(\mathrm{a}_{4}-\mathrm{a}_{3}\right), \mathrm{a}_{6}-\left(\frac{\alpha-\mathrm{k}}{1-\mathrm{k}}\right)\left(\mathrm{a}_{6}-\mathrm{a}_{5}\right)\right]} & ; \alpha \in(\mathrm{k}, 1]
\end{array}\right\}
$$

\section{Two Person Zero Sum Fuzzy Game}

- $\quad$ Two person (player) zero sum fuzzy game is denoted by $F G \approx\left(\widetilde{\mathrm{S}}_{1}, \widetilde{\mathrm{S}}_{2}, \widetilde{\mathrm{K}}, \widetilde{\mathrm{A}}\right)$ where

- $\quad \tilde{\mathrm{S}}_{1} \approx\left\{\widetilde{\mathrm{X}} \approx\left(\tilde{\mathrm{x}}_{1}, \tilde{\mathrm{x}}_{2}, \tilde{\mathrm{x}}_{3}, \ldots, \tilde{\mathrm{x}}_{\mathrm{m}}\right) \mid \tilde{\mathrm{x}}_{\mathrm{i}} \geq \tilde{0}, \forall \mathrm{i}=1,2,3, \ldots \mathrm{m}, \sum_{\mathrm{i}=1}^{\mathrm{m}} \tilde{\mathrm{x}}_{\mathrm{i}} \approx \tilde{\mathrm{I}}\right\}$

- $\quad \tilde{S}_{2} \approx\left\{\widetilde{Y} \approx\left(\tilde{y}_{1}, \tilde{y}_{2}, \tilde{y}_{3}, \ldots ., \tilde{y}_{n}\right) \mid \tilde{y}_{j} \geq \tilde{0}, \forall j=1,2,3, \ldots n, \sum_{j=1}^{n} \tilde{y}_{j} \approx \tilde{I}\right\}$

- Where $\tilde{S}_{1}$ and $\tilde{S}_{2}$ are strategic spaces available for both player I and II respectively. Then the fuzzy payoffs gained by maximizing player is defined as,

- $\quad \widetilde{\mathrm{K}}(\widetilde{\mathrm{X}}, \widetilde{\mathrm{Y}})=\sum_{\mathrm{i}=1}^{\mathrm{m}} \sum_{\mathrm{j}=1}^{\mathrm{n}} \tilde{\mathrm{a}}_{\mathrm{ij}} \cdot \tilde{\mathrm{X}}_{\mathrm{i}} \cdot \tilde{\mathrm{y}}_{\mathrm{j}} \approx \widetilde{\mathrm{X}}^{\mathrm{T}} \cdot \widetilde{\mathrm{A}} \cdot \widetilde{\mathrm{Y}}$

\section{Existing Ranking Methods to Convert OFN into Crisp Value}

Let $\tilde{A}$ be an octagonal fuzzy number then different kinds of ranking methods or techniques are used for defuzzification are tabled as:

Table: 4.1 Existing Ranking Methods

\begin{tabular}{|l|l|}
\hline Ranking Method & Formula to Obtain Crisp Value of OFN \\
\hline $\begin{array}{l}\text { Measure of an OFN (Magnitude } \\
\text { ranking method) }\end{array}$ & $\begin{array}{l}\mathrm{M}_{\mathrm{o}}^{\text {oct }}(\widetilde{\mathrm{A}})=\frac{1}{4}\left[\mathrm{k}\left(\mathrm{a}_{1}+\mathrm{a}_{2}+\mathrm{a}_{7}+\mathrm{a}_{8}\right)+(1-\mathrm{k})\left(\mathrm{a}_{3}+\mathrm{a}_{4}+\mathrm{a}_{5}+\right.\right. \\
\mathrm{a} 6 ; \mathrm{k} \in[0,1]\end{array}$ \\
\hline $\begin{array}{l}\text { Pascal's triangular graded mean } \\
\text { method }\end{array}$ & $\mathrm{P}(\widetilde{\mathrm{A}})=\frac{\left(\mathrm{a}_{1}+\mathrm{a}_{8}\right)+7\left(\mathrm{a}_{2}+\mathrm{a}_{7}\right)+21\left(\mathrm{a}_{3}+\mathrm{a}_{6}\right)+35\left(\mathrm{a}_{4}+\mathrm{a}_{5}\right)}{128}$ \\
\hline Simple average method & $\mathrm{P}(\widetilde{\mathrm{A}})=\frac{\mathrm{a}_{1}+\mathrm{a}_{2}+\mathrm{a}_{3}+\mathrm{a}_{4}+\mathrm{a}_{5}+\mathrm{a}_{6}+\mathrm{a}_{7}+\mathrm{a}_{8}}{8}$ \\
\hline Mean-max membership principle & $\mathrm{P}(\widetilde{\mathrm{A}})=\frac{\mathrm{a}_{4}+\mathrm{a}_{5}}{2}$ \\
\hline Centroid method & $\mathrm{P}(\widetilde{\mathrm{A}})=\frac{\int_{\mathrm{a}_{1}}^{\mathrm{a}_{8}} \mathrm{x} \mu_{\widetilde{\mathrm{A}}}(\mathrm{x}) \mathrm{dx}}{\int_{\mathrm{a}_{1}}^{\mathrm{a}} \mu_{\widetilde{\mathrm{A}}}(\mathrm{x}) \mathrm{dx}}$ \\
\hline
\end{tabular}




\begin{tabular}{|c|c|}
\hline First of maxima (FOM) method & $P(\widetilde{\mathrm{A}})=\mathrm{a}_{4}$ \\
\hline Last of maxima (LOM) method & $P(\widetilde{\mathrm{A}})=\mathrm{a}_{5}$ \\
\hline
\end{tabular}

\section{Proposed Ranking Methods to Convert an OFN into Crisp Value}

Let $\widetilde{A}$ be an octagonal fuzzy number then we propose some defuzzified ranking methods namely average of odd positional values in OFN, average of even positional values in OFN, Quartile deviations methods, average of the smallest possible $\alpha$-cut approach for an OFN and average of the largest possible $\alpha$-cut approach for an OFN to obtain a crisp value as defined as follows

\subsection{Average of Odd Positional Values in OFN}

This defuzzification can be expressed as $P(\widetilde{A})=\frac{\left(a_{1}+a_{3}+a_{5}+a_{7}\right)}{4}$

\subsection{Average of Even Positional Values in OFN}

This defuzzification can be expressed as $P(\widetilde{A})=\frac{\left(a_{2}+a_{4}+a_{6}+a_{8}\right)}{4}$

\subsection{Quartile Deviations}

First quartile deviation $\mathrm{P}_{\mathrm{Q}_{1}}(\widetilde{\mathrm{A}})=\left(\frac{\mathrm{n}+1}{4}\right)^{\text {th }}$ observational value in OFN

Second quartile deviation $\mathrm{P}_{\mathrm{Q}_{2}}(\widetilde{\mathrm{A}})=2\left(\frac{\mathrm{n}+1}{4}\right)^{\text {th }}$ observational value in OFN

Third quartile deviation $\mathrm{P}_{\mathrm{Q}_{3}}(\widetilde{\mathrm{A}})=3\left(\frac{\mathrm{n}+1}{4}\right)^{\text {th }}$ observational value in OFN

5.4 Average of the Smallest Possible $\alpha$-Cut Approach for an OFN

The crisp value can be approximated by the average of $\alpha$-Cut of bounded left continuous non-decreasing and non-increasing functions over the interval $[0, \mathrm{k}]$ i.e.

$$
\mathrm{P}(\widetilde{\mathrm{A}})=\frac{1}{2}\left\{\left(\mathrm{a}_{1}+\frac{\alpha}{\mathrm{k}}\left(\mathrm{a}_{2}-\mathrm{a}_{1}\right)\right)+\left(\mathrm{a}_{8}-\frac{\alpha}{\mathrm{k}}\left(\mathrm{a}_{8}-\mathrm{a}_{7}\right)\right)\right\} \text { over } \alpha \in[0, \mathrm{k}] .
$$

5.5 Average of the Largest Possible $\alpha$-Cut Approach for an OFN

The crisp value can be approximated by the average of $\alpha$-Cut of bounded left continuous non-decreasing and non-increasing functions over the interval[ $\mathrm{k}, 1]$ i.e.

$$
\mathrm{P}(\widetilde{\mathrm{A}})=\frac{1}{2}\left\{\left(\mathrm{a}_{3}+\left(\frac{\alpha-\mathrm{k}}{1-\mathrm{k}}\right)\left(\mathrm{a}_{4}-\mathrm{a}_{3}\right)\right)+\left(\mathrm{a}_{6}-\left(\frac{\alpha-\mathrm{k}}{1-\mathrm{k}}\right)\left(\mathrm{a}_{6}-\mathrm{a}_{5}\right)\right)\right\} \text { over } \alpha \in[\mathrm{k}, 1] \text {. }
$$

\section{Application in Marketing}

There are two companies namely, McDonald's and Burger King in market. Both the companies have three strategies viz. Discounted price, status quo and Aggressive commercial. In such situations uncertainty is measure factor. These situations can be modeled efficiently by using ranking functions. In this particular problem we are taking octagonal fuzzy numbers as payoffs of both the companies. Verbal phrases for octagonal fuzzy numbers in terms of profit are,

VVL-Very Very Low,

VL-Very Low, L-Low,

LM-Low Mean,

HM-High Mean,

H-High,

VH-Very High,

VVH-Very Very High 
Table: 6.1 Verbal Phrases for Octagonal Fuzzy Numbers

\begin{tabular}{|c|c|c|c|}
\hline & Discounted price & status quo & Aggressive commercial \\
\hline $\begin{array}{c}\text { Discounted } \\
\text { price }\end{array}$ & $(\mathrm{VVL}, \mathrm{VL}, \mathrm{L}, \mathrm{LM}, \mathrm{HM}, \mathrm{H}, \mathrm{VH}, \mathrm{VVH})$ & $(\mathrm{VVL}, \mathrm{VL}, \mathrm{L}, \mathrm{LM}, \mathrm{HM}, \mathrm{H}, \mathrm{VH}, \mathrm{VVH})$ & (VVL,VL,L,LM,HM,H,VH,VVH) \\
\hline $\begin{array}{c}\text { status quo } \\
\text { Aggressivec } \\
\text { ommercial }\end{array}$ & $(\mathrm{VVL}, \mathrm{VL}, \mathrm{L}, \mathrm{LM}, \mathrm{HM}, \mathrm{H}, \mathrm{VH}, \mathrm{VVH})$ & $(\mathrm{VVL}, \mathrm{VL}, \mathrm{L}, \mathrm{LM}, \mathrm{HM}, \mathrm{H}, \mathrm{VH}, \mathrm{VVH})$ & $(\mathrm{VVL}, \mathrm{VL}, \mathrm{L}, \mathrm{LM}, \mathrm{HM}, \mathrm{H}, \mathrm{VH}, \mathrm{VVH})$ \\
\hline & $(\mathrm{VVL}, \mathrm{VL}, \mathrm{L}, \mathrm{LM}, \mathrm{HM}, \mathrm{H}, \mathrm{VH}, \mathrm{VVH})$ & $(\mathrm{VVL}, \mathrm{VL}, \mathrm{L}, \mathrm{LM}, \mathrm{HM}, \mathrm{H}, \mathrm{VH}, \mathrm{VVH})$ & \\
\end{tabular}

Table: 6.2 Payoff matrix for McDonald's and Burger King

\begin{tabular}{|l|c|c|c|}
\hline & Discounted price & status quo & Aggressive commercial \\
\hline $\begin{array}{l}\text { Discounted } \\
\text { price }\end{array}$ & $(5,6,7,8,9,10,11,12)$ & $(0,1,2,3,4,5,6,7)$ & $(0,1,2,3,4,5,6,7)$ \\
\hline status quo & $(3,4,5,6,7,8,9,10)$ & $(4,5,6,7,8,9,10,11)$ & $(4,5,6,7,8,9,10,11)$ \\
\hline $\begin{array}{l}\text { Aggressive } \\
\text { commercial }\end{array}$ & $(-11,-10,-9,-8,-7,-6,-5,-4)$ & $(1,2,3,4,5,6,7,8)$ & $(-2,-1,0,1,2,3,4,5)$ \\
\end{tabular}

\section{Burger King}

McDonald's $\quad\left[\begin{array}{ccc}(5,6,7,8,9,10,11,12) & (-1,0,1,2,3,4,5,6,) & (0,1,2,3,4,5,6,7) \\ (3,4,5,6,7,8,9,10) & (2,3,4,5,6,7,8,9) & (4,5,6,7,8,9,10,11) \\ (-11,-10,-9,-8,-7,-6,-5,-4) & (1,2,3,4,5,6,7,8) & (-2,-1,0,1,2,3,4,5)\end{array}\right]$

Where

$\tilde{\mathrm{a}}_{11}=(5,6,7,8,9,10,11,12) ; \tilde{\mathrm{a}}_{12}=(-1,0,1,2,3,4,5,6,) ; \tilde{\mathrm{a}}_{13}=(0,1,2,3,4,5,6,7)$

$\tilde{\mathrm{a}}_{21}=(3,4,5,6,7,8,9,10) ; \widetilde{\mathrm{a}}_{22}=(2,3,4,5,6,7,8,9) ; \widetilde{\mathrm{a}}_{23}=(4,5,6,7,8,9,10,11)$;

$\tilde{\mathrm{a}}_{31}=(-11,-10,-9,-8,-7,-6,-5,-4) ; \tilde{\mathrm{a}}_{32}=(1,2,3,4,5,6,7,8) ; \tilde{\mathrm{a}}_{33}=(-2,-1,0,1,2,3,4,5)$ 
Table: 6.3 Best strategies and value of game

\begin{tabular}{|c|c|c|c|}
\hline Defuzzification Method & $\begin{array}{l}\text { Optimal } \\
\text { Strategy } \\
\text { McDonald's }\end{array}$ & \begin{tabular}{l}
\multicolumn{2}{c}{ Optimal } \\
Strategy Burger \\
King
\end{tabular} & $\begin{array}{l}\text { Value of the } \\
\text { Game }\end{array}$ \\
\hline $\begin{array}{l}\text { Measure of an OFN (Magnitude } \\
\text { ranking method) }\end{array}$ & II & II & 5.5 \\
\hline $\begin{array}{l}\text { Pascal's triangular graded mean } \\
\text { method }\end{array}$ & II & II & 5.5 \\
\hline Simple average method & II & II & 5.5 \\
\hline $\begin{array}{l}\text { Mean-max membership principle } \\
\text { (MOM Method) }\end{array}$ & II & II & 5.5 \\
\hline Centroid method & II & II & 5.5 \\
\hline First of maxima (FOM) method & II & II & 5.0 \\
\hline Last of maxima (LOM) method & II & II & 6.0 \\
\hline $\begin{array}{l}\text { Average of Odd Positional Values in } \\
\text { OFN }\end{array}$ & II & II & 5.0 \\
\hline $\begin{array}{l}\text { Average of Even Positional Values in } \\
\text { OFN }\end{array}$ & II & II & 6.0 \\
\hline First Quartile Deviation & II & II & 3.25 \\
\hline Second Quartile Deviation & II & II & 5.5 \\
\hline Third Quartile Deviation & II & II & 7.75 \\
\hline $\begin{array}{l}\text { Average of the Smallest Possible } \alpha \text { - } \\
\text { Cut Approach for an OFN }\end{array}$ & II & II & 5.5 \\
\hline $\begin{array}{l}\text { Average of the Largest Possible } \alpha \text { - } \\
\text { Cut Approach for an OFN }\end{array}$ & II & II & 5.5 \\
\hline
\end{tabular}

If payoff matrix is given by,

McDonald's $\left[\begin{array}{ccc}(-2,-1,0,1,2,3,4,5) & (0,1,2,3,4,5,6,7) & (2,3,4,5,6,7,8,9) \\ (1,2,3,4,5,6,7,8) & (9,10,11,12,13,14,15,16) & (6,7,8,9,10,11,12,13) \\ (8,9,10,11,12,13,14,15) & (-1,0,1,2,3,4,5,6) & (11,12,13,14,15,16,17,18)\end{array}\right]$

Where

\section{Burger King}

$\tilde{\mathrm{a}}_{11}=(-2,-1,0,1,2,3,4,5) ; \tilde{\mathrm{a}}_{12}=(0,1,2,3,4,5,6,7) ; \widetilde{\mathrm{a}}_{13}=(2,3,4,5,6,7,8,9) ; \widetilde{\mathrm{a}}_{21}=(1,2,3,4,5,6,7,8) ;$

$\tilde{\mathrm{a}}_{22}=(9,10,11,12,13,14,15,16) ; \tilde{\mathrm{a}}_{23}=(6,7,8,9,10,11,12,13) ; \widetilde{\mathrm{a}}_{31}=(8,9,10,11,12,13,14,15)$;

$\tilde{\mathrm{a}}_{32}=(-1,0,1,2,3,4,5,6) ; \tilde{\mathrm{a}}_{33}=(11,12,13,14,15,16,17,18)$

Table: 6.4 Best strategies and value of game

\begin{tabular}{|c|c|c|c|}
\hline Defuzzification Method & $\begin{array}{c}\text { Optimal Strategy } \\
\text { McDonald's }\end{array}$ & $\begin{array}{c}\text { Optimal Strategy } \\
\text { Burger King }\end{array}$ & $\begin{array}{c}\text { Value of the } \\
\text { Game }\end{array}$ \\
\hline $\begin{array}{c}\text { Measure of an OFN } \\
\text { Magnitude } \\
\text { method) }\end{array}$ & $(0,0.529,0.470)$ & $(0.588,0.411,0)$ & 7.794 \\
\hline $\begin{array}{c}\text { Pascal’s triangular } \\
\text { graded mean method }\end{array}$ & $(0,0.529,0.470)$ & $(0.588,0.411,0)$ & 7.794 \\
\hline Simple average method & $(0,0.529,0.470)$ & $(0.588,0.411,0)$ & 7.794 \\
\hline $\begin{array}{c}\text { Mean-max membership } \\
\text { principle (MOM Method) }\end{array}$ & $(0,0.529,0.470)$ & $(0.588,0.411,0)$ & 7.794 \\
\hline $\begin{array}{c}\text { Centroid method } \\
\text { First of maxima (FOM) } \\
\text { method }\end{array}$ & $(0,0.529,0.470)$ & $(0.588,0.411,0)$ & 7.294 \\
\hline $\begin{array}{c}\text { Last of maxima (LOM) } \\
\text { method }\end{array}$ & $(0,0.529,0.470)$ & $(0.588,0.411,0)$ & 8.294 \\
\hline
\end{tabular}




\begin{tabular}{|c|c|c|c|}
\hline $\begin{array}{c}\text { Average of Odd } \\
\text { Positional Values in OFN }\end{array}$ & $(0,0.529,0.470)$ & $(0.588,0.411,0)$ & 7.294 \\
\hline $\begin{array}{c}\text { Average of Even } \\
\text { Positional Values in OFN }\end{array}$ & $(0,0.529,0.470)$ & $(0.588,0.411,0)$ & 8.294 \\
\hline First Quartile Deviation & $(0,0.529,0.470)$ & $(0.588,0.411,0)$ & 5.544 \\
\hline $\begin{array}{c}\text { Second Quartile } \\
\text { Deviation }\end{array}$ & $(0,0.529,0.470)$ & $(0.588,0.411,0)$ & 7.794 \\
\hline Third Quartile Deviation & $(0,0.529,0.470)$ & $(0.588,0.411,0)$ & 10.044 \\
\hline $\begin{array}{c}\text { Average of the Smallest } \\
\text { Possible } \alpha \text {-Cut Approach } \\
\text { for an OFN }\end{array}$ & $(0,0.529,0.470)$ & $(0.588,0.411,0)$ & 7.794 \\
\hline $\begin{array}{c}\text { Average of the Largest } \\
\text { Possible } \alpha \text {-Cut Approach } \\
\text { for an OFN }\end{array}$ & $(0,0.529,0.470)$ & $(0.588,0.411,0)$ & 7.794 \\
\hline
\end{tabular}

\section{Graphical Representation}

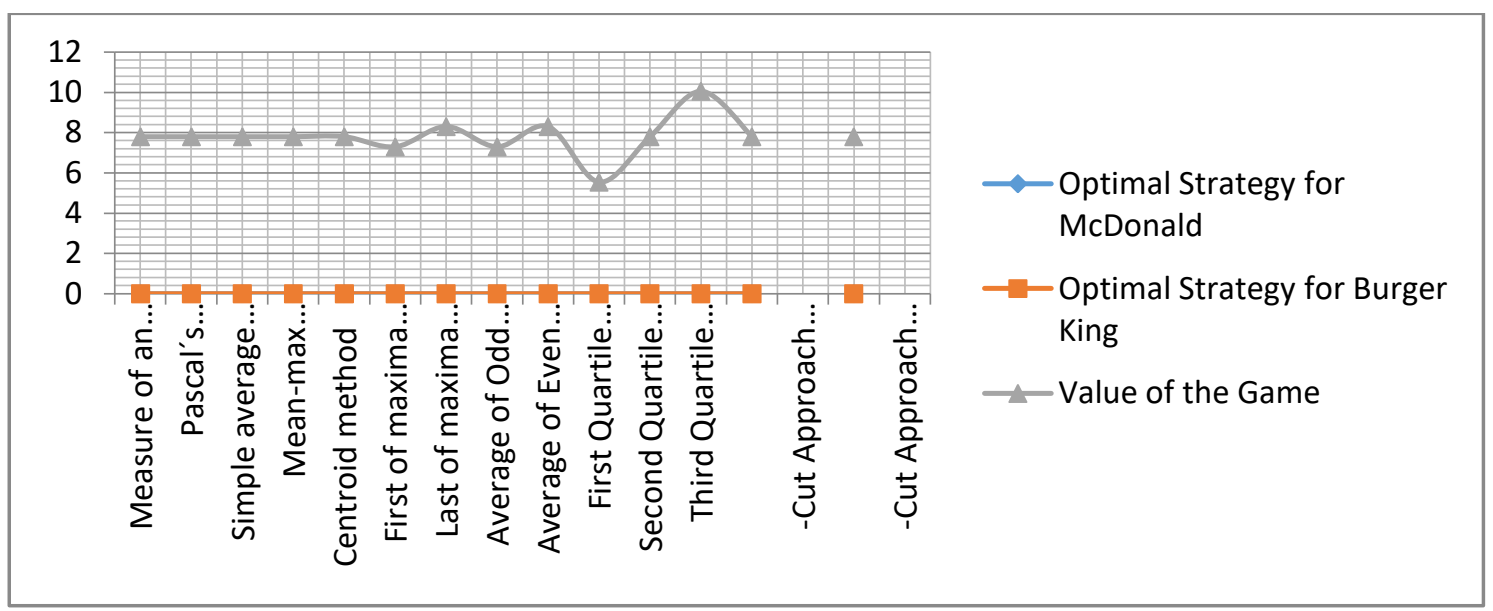

Figure: 7.1 Best strategies and value of game

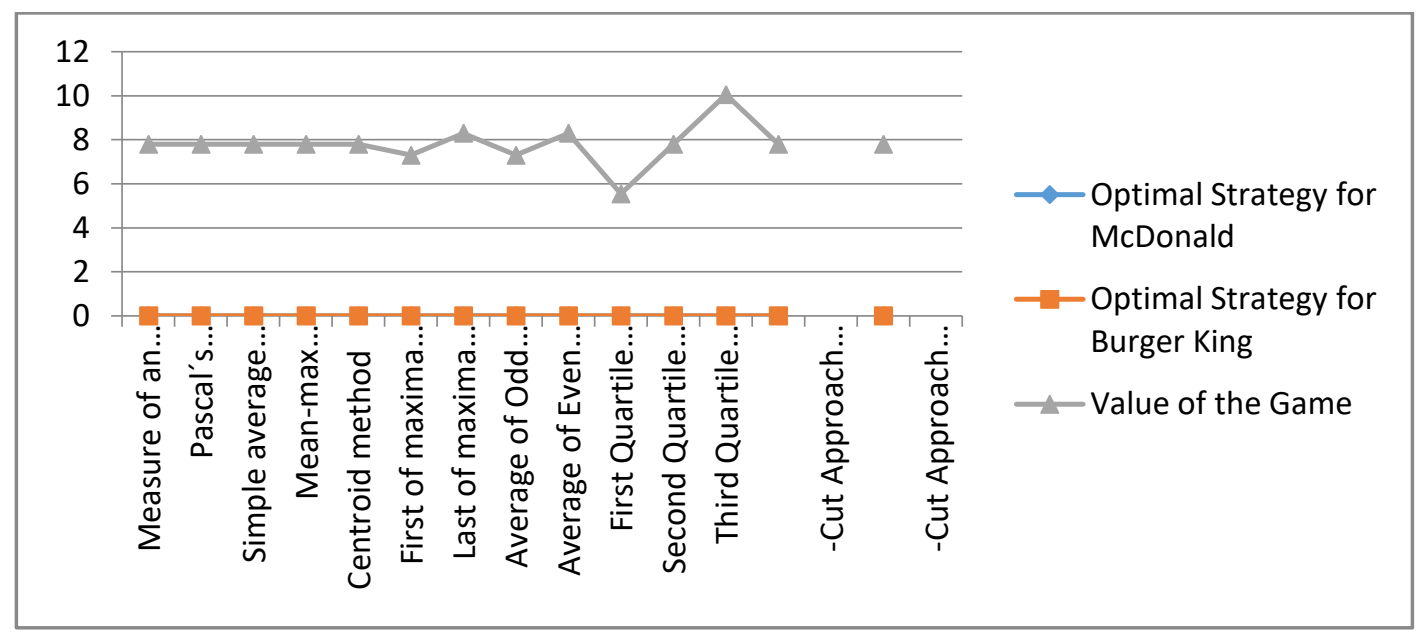

Figure: 7.2 Best strategies and value of game 


\section{Conclusion}

It is established that competitive situations of marketing can be modeled in form of fuzzy matrix games. By solving matrix games, best strategies and optimal value can be obtained. In the present paper, an approach for solving octagonal fuzzy matrix game problem is proposed. It is established that our results are same as by using different kinds of techniques like as ranking for a fuzzy number, Pascal's triangular graded mean formula, simple average method, centroid method, FOM, LOM, MOM methods, odd, and even positional values methods, quartile deviation methods, and $\alpha$-cut approach. Decision maker can suitably modify the parameter $\mathrm{k}$ to get the desired result. Different fuzzy game values yields for the same fuzzy game corresponding to distinct values of the parameter k.

\section{References (APA)}

1. Abouzar S. and Sayed M. Karbassi ( 2018). Solving multi-objective transportation problems with fuzzy objective functions coefficients using relations between intervals and a-cut set of a fuzzy number. ADVANCES AND APPLICATIONS IN MATHEMATICAL SCIENCES, 18 (2), 197-261.

2. Bortolan, G. and Degani, R. (1985). A review of some method for ranking fuzzy sets, Fuzzy sets and systems. 15, 1-19.

3. Butnariu D., (1978). Fuzzy games: A description of the Concept, Fuzzy sets and systems, 1(3), 181-192.

4. Cevikel, A.C. and Ahlatciuglu, M., (2009). A new solution concept in fuzzy matrix games. World applied sciences journal, 7(7), 866-871.

5. Christi, M.S.A. and Kalpana, N., (2016). Fuzzy game matrix solutions using average weighted approach. International journal of multi-disciplinary research review, 1(20), 151160.

6. Delgado, M., Verdegay, J.L. and Vila, M.A., (1988). A procedure for ranking fuzzy numbers. Fuzzy sets and systems, 26, 49-62.

7. Liou, T.S. and Wang, M.J., (1992). Ranking fuzzy numbers with integral value. Fuzzy sets and systems, 50(3), 247-255.

8. Malini, P. and Ananthanarayanan, A., (2016). Solving fuzzy transportation problems using ranking of octagonal fuzzy numbers. International journal of pure and applied mathematics, 110(2), 275-282.

9. Malini, S. U. and Kennedy, F. C., (2013). An approach for solving transportation problem using octagonal fuzzy numbers. Applied mathematical sciences. 7, 2661-2673.

10. McGuire T. W. and Staelin R., (1983). An Industry Equilibrium Analysis of Downstream Vertical Integration. Marketing Science, 2(2), 161-191.

11. Milgrom P. and Roberts J., (1986). Price and Advertising Signals of Product Quality. Journal of Political Economy, 94(4), 796-821.

12. Mitchell T. J. and Hustad T. P., (1981). The Analysis of Options Approach to New Product Screening. Research in Marketing, 4, 143-179.

13. Nadia Z. and Abolfazl A. Pishkoohi, (2018). A modern approach for intelligent intersection traffic control with fuzzy logic. ADVANCES AND APPLICATIONS IN MATHEMATICAL SCIENCES, 17(6), 429-502.

14. Sharma, S. C. and Kumar, G., (2016). A computational approach to solve games with uncertainty and an applicatioan in election forecasting. Jananabha, 46, 75-86.

15. Yuan, Y., (1991). Criteria for evaluating fuzzy ranking methods. Fuzzy sets and systems, 43(2), 139-157.

16. Zadeh, L.A., (1965). Fuzzy sets. Information and Control, 8(3), 338-353. 